\title{
Development of optical systems for the KLYPVE experiment
}

\section{S. Sharakin, ${ }^{a}$ Y. Takizawa, ${ }^{b}$ S.Biktemerova ${ }^{c}$ P. Klimov, ${ }^{a}$ M. Panasyuk ${ }^{* a d}$ and N. Sakaki, ${ }^{b}$ for the JEM-EUSO Collaboration}

${ }^{a}$ D.V. Skobeltsyn Institute of Nuclear Physics, M.V. Lomonosov Moscow State University, Moscow 119991, Russia

${ }^{b}$ RIKEN, 2-1 Hirosawa, Wako, 351-0198 Japan

${ }^{c}$ Joint Institute of Nuclear Research, Dubna, Russia

${ }^{d}$ Faculty of Physics, M.V. Lomonosov Moscow State University, Moscow 119991, Russia

E-mail: sharakin@mail.ru, takkyepostman.riken.jp,

sveta.biktemerova@gmail.com, pavel.klimov@gmail.com,

panasyuk@sinp.msu.ru, sakaki@sci.osaka-cu.ac.jp,

KLYPVE is an orbital detector of ultra high energy cosmic rays to be deployed on the Russian Segment of the International Space Station. An important part of the detector, which determines its physical parameters (energy threshold, field of view) is the optical system. For the project, a two-component system composed of a large area mirror-concentrator and a correcting Fresnel lens was developed. Two options were considered: a "Baseline" and a "Multieye telescope system" (METS). The first one consists of a $3.4 \mathrm{~m}$ diameter mirror and a $1.7 \mathrm{~m}$ lens and has $\pm 14^{\circ}$ field of view. The second one consists of three identical telescopes with $\pm 10^{\circ}$ field of view (a mirror of $2.4 \mathrm{~m}$ diameter and a $1.2 \mathrm{~m}$ lens) and can operate in various modes. The production of a mold for the central segment of the METS mirror was done in SINP MSU. This mold will be used for a carbon-plastic mirror manufacture. It is an important step in the development of lightweight and space qualified optical systems with high performance parameters production technology. A detailed description of the developed optical systems, optimization studies and simulations are presented as well as results of the mold production and first steps in carbon plastic mirror manufacturing.

The 34th International Cosmic Ray Conference,

30 July-6 August, 2015

The Hague, The Netherlands

\footnotetext{
* Speaker.
} 


\section{Introduction}

The observation of the ultra high cosmic rays (UHECR, E $>50 \mathrm{EeV}$ ) from space is one of the most prospective method of future UHECRs measurements, because it allows to achieve a huge field of view (FOV), more than any ground based observatory, and uniform exposition over the celestial sphere (which is also impossible while measuring from ground). Several orbital UHECR detectors are being developed nowadays: TUS and KLYPVE with a reflective optical systems (OS) and JEM-EUSO with even wider FOV system of Fresnel lenses [1].

Detector TUS will be the first space mission, devoted to UHECRs studies and launched on board Lomonosov satellite [2]. This detector has a simple structure (one mirror and the photo receiver with 256 pixels in the focal plane) and rather narrow FOV $\left( \pm 4.5^{\circ}\right)$. The main purpose of the detector is to prove the method of measurements of UHECR from space collecting fluorescent light emitted by extensive air shower in the atmosphere. The OS of the TUS detector is a Fresnel mirror with $\sim 2 \mathrm{~m}^{2}$ area and focal distance of $1.5 \mathrm{~m}$. It is produced by Space Regata Consortium and will be the first sample of light weighted carbon-plastic mirror in space.

\section{KLYPVE optical system}

In 2012, Skobeltsyn Institute of Nuclear Physics of Moscow State University finished the preliminary design stage of the KLYPVE ${ }^{1}$ reflector type telescope for UHECR measurements from the ISS. The main component of the detector is a segmented optical system (OS) with a large entrance pupil. All parts of the telescope are to be delivered to the Russian segment of the ISS by the Progress-TM vehicle.

The main goal of KLYPVE is to considerably, up to $\pm 7.5^{\circ}$, increase the field of view (FOV) of the detector in comparison with TUS and to decrease the energy threshold. The mirror-concentrator has a reflective surface of about $10 \mathrm{~m}^{2}$ (entrance pupil diameter $3.6 \mathrm{~m}$, see figure 1). The focal distance of the system is $3 \mathrm{~m}$ so the angular resolution of the OS is the same as in the TUS detector, i.e. $5 \mathrm{mrad}$ (pixel size $15 \mathrm{~mm} \times 15 \mathrm{~mm}$ ).

However, it became clear during the preliminary design phase that the characteristics of the instrument (observation area and image quality) do not allow solving the problems related to anisotropy of EECRs. The main reason for that is a large off-axis aberration of such fast optical system. Furthermore for an accurate reconstruction of EECR parameters (primary energy and direction), it is desirable to make a pixel of the photodetector smaller than $15 \mathrm{~mm}$. Thus one has to look for optics with a much smaller spot size over the whole FOV. These considerations initiated the development of a new OS for the KLYPVE detector in order to increase the FOV and to improve the spatial and angular resolution and the overall performance of the instrument. This work began in a close collaboration with members of the JEM-EUSO Collaboration in late 2013.

Several options were considered for OS improvements including Fresnel mirror with curved gross surface (like Davies-Cotton system and its generalisations [3]). Detailed simulation showed that it is not possible to achieve a good optical performance for field angles greater than $7^{\circ}$ with a concentrator only. So to eliminate the off-axis aberration an additional corrective element was introduced into the telescope system in form of a Fresnel lens.

\footnotetext{
${ }^{1}$ KLYPVE is a Russian acronym for extreme energy cosmic rays.
} 


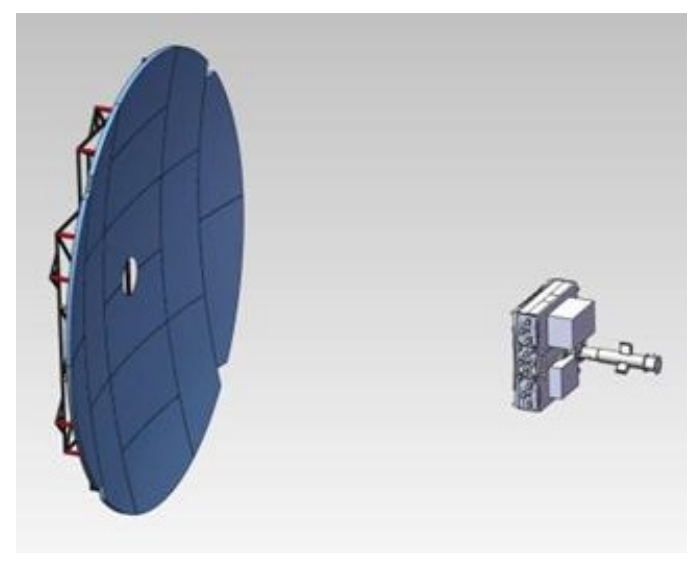

Figure 1: KLYPVE segmented mirror-concentrator and photodetector. One variant of the supporting frame is shown at the rear side of the mirror.

A radial Fresnel structure of the lens has grooves with depth $1 \mathrm{~mm}$ and width varying from several centimeter at the center to about $1 \mathrm{~mm}$ at the edge of the lens. The thickness of the lens should be sufficiently small, about $1 \mathrm{~cm}$. The material of the lens is UV-transparent version of polymethyl metacrylate PMMA-000 (by Mitsubishi Rayon Co., Ltd.). It has a good UV transmittance (more than $95 \%$ for $10 \mathrm{~mm}$ thickness layer at wavelength more than $320 \mathrm{~nm}$ ), with a refractive index of about 1.51. Lenses with similar characteristics were manufactured at Ohmori Materials Fabrication Laboratory, RIKEN (Japan) in 2009-2011 [4].

Depending on the size and complexity of the forms of individual optical elements of the system, two options have been proposed.

\section{Baseline system}

In the so called Baseline system, the diameter of the reflector and the lens-corrector are quite large, $3.4 \mathrm{~m}$ and $1.7 \mathrm{~m}$ respectively. The total length of the system (more precisely, the axial distance from the mirror vertex to the center of the focal surface) is equal to $4 \mathrm{~m}$, the distance from the lens to the focal surface equals $70 \mathrm{~cm}$, see figure 2. In this case, it is possible to expand the FOV up to $\pm 14^{\circ}$, and the RMS-diameter of the image is not larger than $6 \mathrm{~mm}$ in the entir FOV. In this case the angular resolution of the system is $\approx 0.057^{\circ}$, which is equivalent to $\sim 0.4 \mathrm{~km}$ at ground.

One disadvantage of this approach is the difficulty of manufacturing of optical elements, especially the lenses. To correct aberrations over the entire FOV both lens surfaces should have a small curvature (spherical with a radius $1872 \mathrm{~cm}$ ), the front side is a Fresnel surface (with a radial structure of grooves of $1 \mathrm{~mm}$ depth), and the back side is a diffraction surface (with grooves depth $700 \mathrm{~nm}$, see the right side of figure 2). All system parameters were obtained due the optimisation in Code V simulation model.

Two main optical performance characteristics, the spot size and optical efficiency, have been determined by non-sequential ray tracing for Baseline system. Table 1 represents these parameters at different field angles as RMS diameter $d$ of the focal surface image and as the ratio of the number of rays in the spot to the number of rays at the entrance pupil. 

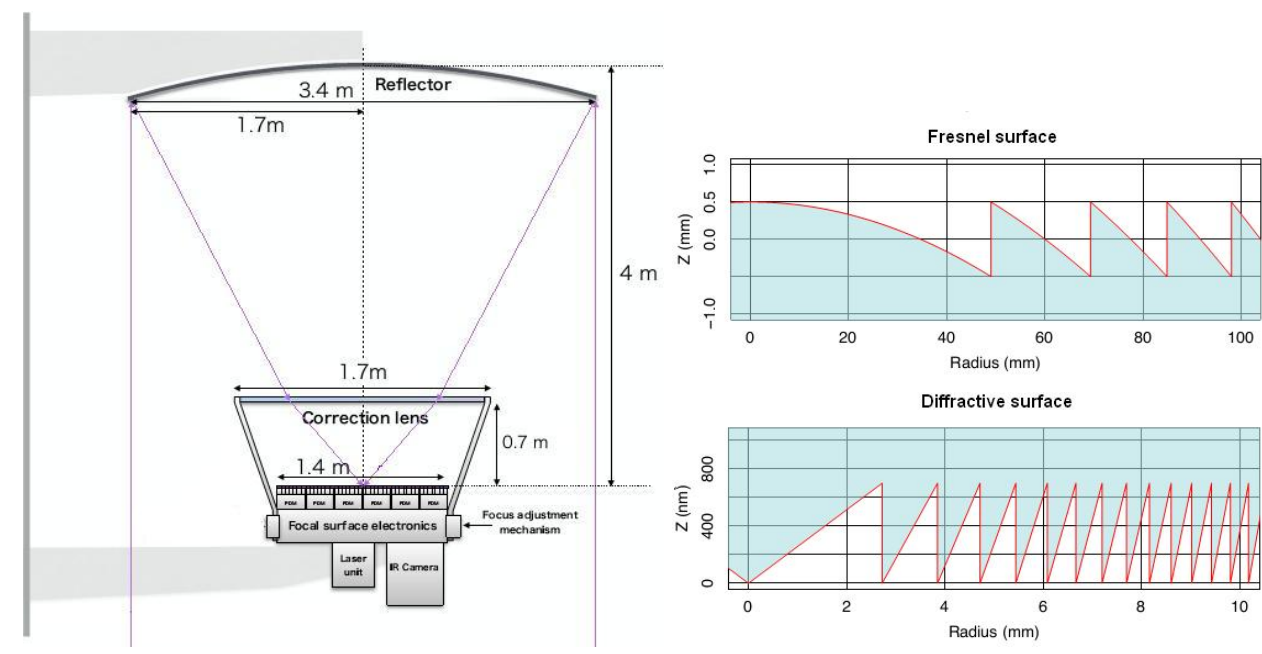

Figure 2: The optics of the Baseline system. Right: grooves of the Fresnel and diffractive structures of the corrective lens.

Table 1: Baseline OS performance characteristics for different field angles.

$\begin{array}{lllllllll}\text { Field Angle } & 0^{\circ} & 4^{\circ} & 6^{\circ} & 8^{\circ} & 10^{\circ} & 12^{\circ} & 13^{\circ} & 14^{\circ} \\ \text { Spot Size, mm } & 2.8 & 2.6 & 3.2 & 4.2 & 4.5 & 4.3 & 4.0 & 3.9 \\ \text { Optical Efficiency, \% } & 62.8 & 61.7 & 58.8 & 57.4 & 51.4 & 43.8 & 37.1 & 29.6\end{array}$

Delivering such a huge system to the ISS is a complicated task by itself. If a Progress-TM is used, the cargo must be first placed inside the ISS and thus pieces of the instrument have to pass through a cylindrical lock of $70 \mathrm{~cm}$ diameter and $120 \mathrm{~cm}$ length. The currently offered solutions require segmentation of all the major components of the system, including the lens, the mirror and the photodetector, and subsequent deployment in space.

\section{Multi-Eye Telescope System}

The KLYPVE OS is a wide-field large aperture and fast optics system. For such a complex instrument another option might be a Multi-Eye Telescope System (METS). The main idea of METS approach is to divide a wide FOV into several FOVs of identical smaller telescopes. In this case we have a simplification of an individual telescope so the new system has several advantages: 1) smaller dimensions of individual telescopes and their narrower FOVs allow to eliminate off-axis aberrations without the use of a complex surfaces corrector (diffraction, curved Fresnel); 2) not only manufacturing of the mirror segments and lenses but testing and adjustment of the individual components and the overall system might be simpler; 3) dimensions of the elements (mirror, lens, focal surface) can be chosen in accordance with the technical requriments, so it is possible to transport the individual telescopes assembled.

In the optimization calculations, the following dimensions of each telescope were obtained: diameters of the mirror, lens and focal surface are $2.4 \mathrm{~m}, 1.2 \mathrm{~m}$ and $0.9 \mathrm{~m}$, the total axial length 
$3 \mathrm{~m}$. Correction of aberrations in the FOV $\pm 10^{\circ}$ (which corresponds to the effective diameter of overall FOV of three telescopes $\sim 35^{\circ}$ ) can be achieved using flat Fresnel surfaces only. The angular resolution of METS is $\approx 0.075^{\circ}$, which is equivalent to $\sim 0.5 \mathrm{~km}$ at ground.

Spot size and optical efficiency of the METS telescope are presented in table 2. The sharp increase of the spot size on the edge of the FOV is due to using flat focal surface. To control spots within $5 \mathrm{~mm}$, one should slightly bend the focal surface at the edges.

Table 2: The METS OS performance characteristics for different field angles.

$\begin{array}{lllllllll}\text { Field Angle } & 0^{\circ} & 3^{\circ} & 5^{\circ} & 6^{\circ} & 7^{\circ} & 8^{\circ} & 9^{\circ} & 10^{\circ} \\ \text { Spot Size, mm } & 2.0 & 2.7 & 3.7 & 3.7 & 4.0 & 4.2 & 4.5 & 5.4 \\ \text { Optical Efficiency, \% } & 68.8 & 67.2 & 62.0 & 57.7 & 55.0 & 53.3 & 49.1 & 41.9\end{array}$

The METS in principal has the ability to use an active configuration. A special dynamic support structure of three telescopes can afford one to implement operation of the detector in various modes. In the basic mode the FOVs of individual telescopes are adjacent (or slightly overlapping for cross-calibration). In the coincidence mode (overlapping FOVs), one can significantly reduce the energy threshold. Main drawback and a big challenge to detector's electronic system in this case is the necessity of a special trigger working for all three telescopes in parallel. Finally, in the tilted mode (FOVs of two or all telescopes are inclined relative to the direction at the nadir) it is possible to increase the exposure, and hence to collect larger statistics of the EECR events.

\section{JEM-EUSO telescope-refractor}

JEM-EUSO (the Extreme Universe Space Observatory on board the Japanese Experiment Module [5]) is a system of a UV telescope and an atmospheric monitoring system, which should be installed on Japan Experimental Module of the ISS. The telescope-refractor consists of three Fresnel lenses with an optical aperture of $4.5 \mathrm{~m}^{2}$ and a focal surface detector formed by 137 photodetector modules composed of $\sim 5000$ multi-anode photo-multiplier tubes in total. The focal surface detector thus includes $\sim 3 \cdot 10^{5}$ channels providing a spatial resolution $\approx 0.074^{\circ}$, equivalent to $\sim 0.5 \mathrm{~km}$ at ground. The opening angle of the telescope equals $60^{\circ}$ providing an observational area of $\sim 1.4 \cdot 10^{5} \mathrm{~km}^{2}$ in nadir mode.

The annual exposure of JEM-EUSO for EECRs above $100 \mathrm{EeV}$ is estimated to be an order of magnitude larger than that of Auger [6]. From ISS orbit JEM-EUSO is able to survey the entire celestial sphere almost uniformly. Detailed simulations show that due to these factors JEM-EUSO will be able to detect significant anisotropies of EECRs practically in all feasible astrophysical scenarios thus providing a huge step towards finding sources of EECRs.

Nevertheless in December 2013, the Japanese Space Agency (JAXA) did not select the instrument for deployment on board the JEM. It has been suggested during 17-th JEM-EUSO meeting that refractor system can be presented as an Advanced option of KLYPVE project. But in this case the telescope parameters should be tuned to satisfy new technical requrements.

The optics of JEM-EUSO refractor consists of the following parts [7]: front lens (the one facing space) - curved doublet Fresnel lens (radius of curvature $340.1 \mathrm{~cm}$ ); stop (iris), needed to define 
on axis entrance pupil diameter; middle lens - with Difractive and Fresnel flat surfaces, it serves as field lens (to reduce vignetting factor) and as chromatic corrector; rear lens - curved doublet Fresnel lens (radius of curvature $469.1 \mathrm{~cm}$ ), which focuses light on the focal surface. Thickness of each lens is $10 \mathrm{~mm}$ and they are made of a PMMA-000.

\section{OS comparison}

Figure 3 shows the dependence of the effective area $S_{\text {eff }}$ of the Baseline and METS optical systems of the modified KLYPVE detector and the JEM-EUSO telescope on the field angle $\gamma$. Here, the effective area means the ratio of the luminous flux to irradiance, i.e.,

$$
S_{\text {eff }}=\varepsilon_{\text {opt }}(\gamma) S_{\text {geom }}\left(0^{\circ}\right) \cos \gamma
$$

where $\gamma$ is the field angle, $\varepsilon_{\text {opt }}$ is the optical efficiency, and the axial geometrical area (the second factor) takes into account the reduction of the entrance pupil due to the central screening by the lens. Thus, this parameter is one of the most important in the design of optical systems of an orbital detector.

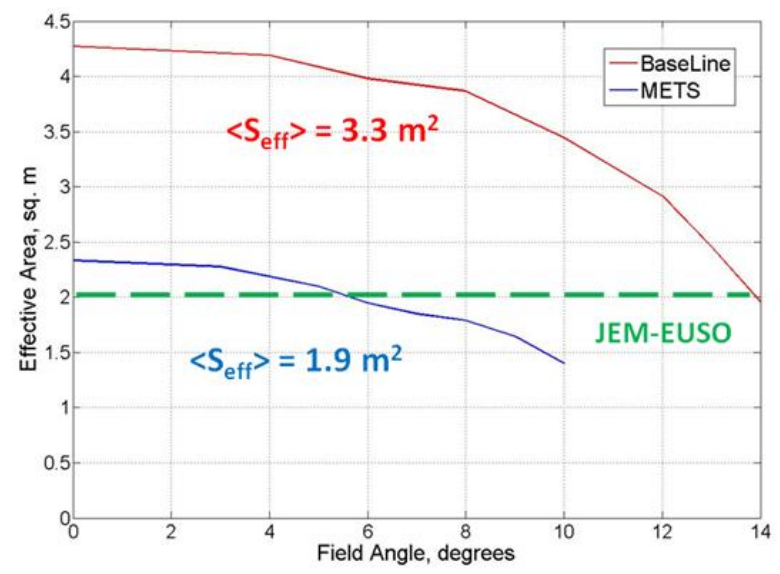

Figure 3: Effective area of the Baseline system and METS compared to JEM-EUSO for the entire FOV. $\left\langle S_{\text {eff }}\right\rangle$ denotes average values of the effective area for the isotropic distribution of light.

The effective area is reduced at the edge of FOV for both systems, primarily because peripherial rays miss the lens at large field angle (trade-off between lens diameter and optic efficiency). The additional loss of efficiency is due to light scattering on Fresnel and diffraction grooves, which leads to a significant spot smearing at large field angles. As can be seen from figure 3 , the effective area of the METS telescope approximately coincides with the JEM-EUSO one. In the case of the Baseline system, it is possible to increase the value of this parameter in average over the entire FOV by $70 \%$, and more than twice for events in the cone $\gamma<10^{\circ}$.

However, it should be noted that a refraction efficiency of the (first) diffraction peak was assumed to be $95 \%$ in these calculations. Reaching such values over the entire surface during manufacturing of the lens can pose a problem since the diffractive surface area is about $2.3 \mathrm{~m}^{2}$. 
Table 3: Performance characteristics (spot size, effective area, FOV and throughput) for 5 different orbital detectors of EECRs.

\begin{tabular}{|l|llll|}
\hline & $d, \mathrm{~mm}$ & $S_{\text {eff }}, \mathrm{m}^{2}$ & $\Omega, \mathrm{sr}$ & $\Omega S_{\text {eff }}, \mathrm{m}^{2} \mathrm{sr}$ \\
\hline JEM-EUSO & 3 & 2 & 0.8 & 1.6 \\
TUS & 20 & 1.2 & 0.025 & 0.03 \\
KLYPVE (orig.) & 20 & 5 & 0.05 & 0.25 \\
Baseline & 3.5 & 3.3 & 0.2 & 0.7 \\
METS & 4 & 1.9 & 0.3 & 0.6 \\
\hline
\end{tabular}

Thus, having undoubted advantages in decreasing the energy threshold, the Baseline system might have the effective FOV much smaller than the total FOV of the METS telescope.

Table 3 summarizes the main characteristics of the five optical systems: the lens system of JEM-EUSO, TUS Fresnel mirrors, the original version of the KLYPVE detector (without any corrector) and the two advanced options, the Baseline system and METS (with three identical telescopes in the basic mode of operation). Scattering of light by Fresnel surfaces was taken into account when calculating the effective area.

\section{Manufacturing and testing of OS elements}

During the 2015 year the MSU part of collaboration started tecnological work on manufacturing mirror mold and carbon plastic mirror. We produced the mold of the central part of the METS mirror (see fig. 4), which has the trapezoidal form with sizes $120 \times 60 \times 60 \times 60 \mathrm{~cm}$. The main goal is to check the possibility of the SINP MSU workshop to mill the mold of sufficient accuracy on the machine DATRON ML 1500-2C. We used a parabolic profile to facilitate subsequent testing of the mold and mirror segments, that will be produced using it.

Parallel to this work we developed the production technology of carbon plastic (CP) mirrors. For that we produce several small-sizes mirror samples with 3-layer construction (two CP layers and aluminium honeycomb between them, see fig. 5). In this production scheme we want to compare the influence on the mirror quality of different tecnological parameters: different $\mathrm{CP}$ tissue texture, different thickness of the CP layers and honeycomb structure, the curvature and quality of the mold, different temperatures and pressure regimes. For this puporse we have manufactured one flat sample, two spherical ones (right part of fig. 5) and one with parabolic profile (on the of-axis part of the trapezoidal mold).

Currently we have started optical measurements of the mold and samples: evaluating their roughness, profile and optical performance characteristics. The results of these first tests will be published elsewhere soon.

\section{Acknowledgments}

The work of Russian co-authors was partially supported by RFBR, research project No. 1302-12175 ofi_m. We would like to thank our colleagues from the workshop of SINP MSU and 


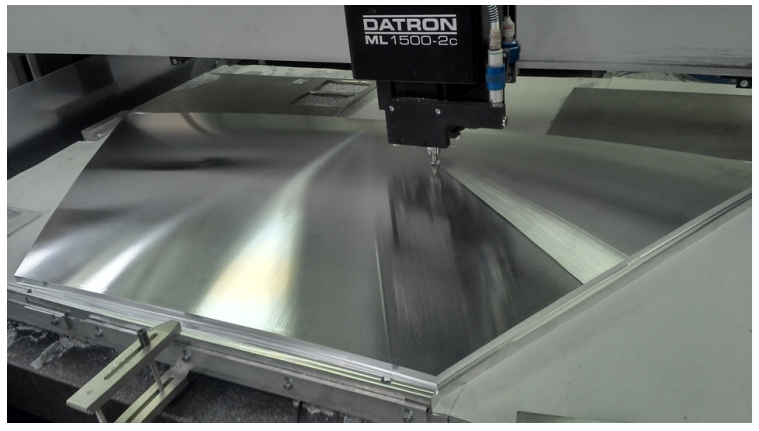

Figure 4: Mold manufacturing for central part of the METS mirror.
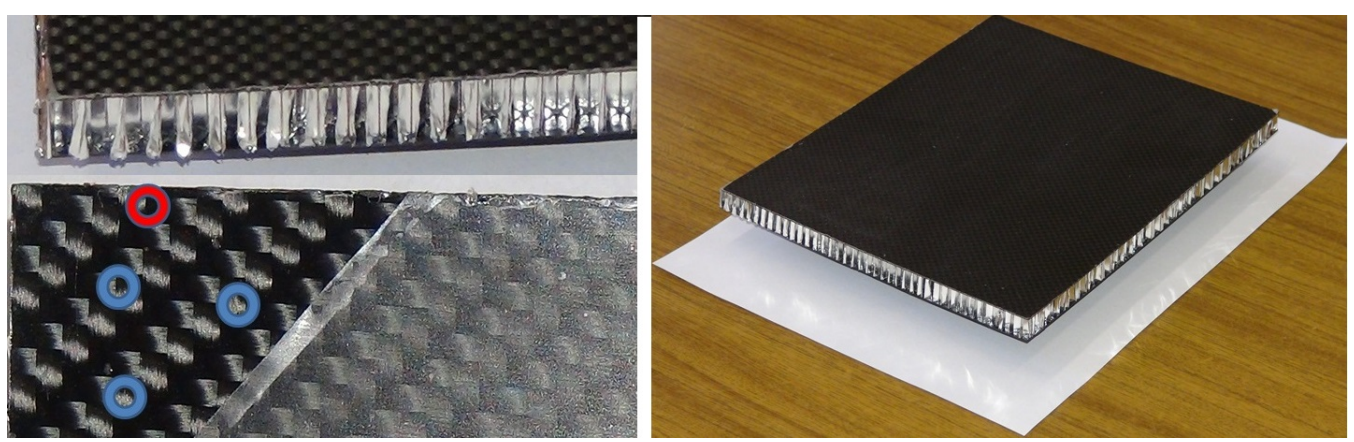

Figure 5: Samples of carbon plastic mirror. Left: flat sample, on top - three layer construction, on bottom - edge of the sample with 4 "points" for roughness estimations (reflective surface partially covered by protective film). Right: sperical sample with sizes $18 \times 20 \mathrm{~cm}^{2}$ and radius of curvature $741 \mathrm{~cm}$.

from Space Regata Consortium (and especcialy A.Pakhomov and A.Senkovskii) for the fruitfull work on mold and carbon plastic mirror manufacturing.

\section{References}

[1] M. Pallavicini, R. Pesce, A. Petrolini, and A. Thea, The observation of extensive air showers from an Earth-orbiting satellite, Astropart. Phys. 35 (Feb., 2012) 402-420.

[2] B. A. Khrenov et al., Pioneering space based detector for study of cosmic rays beyond GZK limit, in European Physical J. Web of Conf., vol. 53, p. 9006, June, 2013.

[3] S. A. Sharakin et al., Mirror-concentrator for space telescope with wide field of view and "high" angular resolution for observation of ultrahigh energy cosmic rays and other atmospheric flashes, in Proceedings of the SPIE, vol. 8443, 2012.

[4] Y. Hachisu et al., JEM-EUSO lens manufacturing, in Proc. 32nd Int. Cosmic Ray Conf. (Beijing), vol. 3, p. 184, 2011.

[5] P. Picozza for the JEM-EUSO collaboration, The JEM-EUSO Program, in Proc. 34th Int. Cosmic Ray Conf. , ID0694, 2015.

[6] J.H. Adams et al., An evaluation of the exposure in nadir observation of the JEM-EUSO mission, Astropart. Phys. 44 (2013) 76-90.

[7] A. Zuccaro Marchi, Y. Takizawa, Y. Takahashi, The JEM-EUSO optics design, in Proc. 32nd Int. Cosmic Ray Conf. (Beijing) 3, p. 180, 2011. 